\title{
Importance of Skin Changes in the Differential Diagnosis of Congenital Muscular Dystrophies
}

\author{
Uluç Yis, ${ }^{1}$ Figen Baydan, ${ }^{2}$ Mert Karakaya, ${ }^{3}$ Semra Hız Kurul, ${ }^{1}$ and Sebahattin Cirak ${ }^{3,4,5}$ \\ ${ }^{1}$ Division of Child Neurology, Department of Pediatrics, School of Medicine, Dokuz Eylül University, 35340 İzmir, Turkey \\ ${ }^{2}$ Izmir Tepecik Training and Research Hospital, Division of Child Neurology, 35040 Izmir, Turkey \\ ${ }^{3}$ University Hospital of Cologne, Institute of Human Genetics, 50931 Cologne, Germany \\ ${ }^{4}$ University Hospital of Cologne, Department of Pediatrics, 50931 Cologne, Germany \\ ${ }^{5}$ Center for Molecular Medicine, 50931 Cologne, Germany
}

Correspondence should be addressed to Sebahattin Cirak; sebahattin.cirak@uk-koeln.de

Received 31 December 2015; Revised 13 March 2016; Accepted 15 March 2016

Academic Editor: Terry K. Smith

Copyright (c) 2016 Uluç Yis et al. This is an open access article distributed under the Creative Commons Attribution License, which permits unrestricted use, distribution, and reproduction in any medium, provided the original work is properly cited.

\begin{abstract}
Megaconial congenital muscular dystrophy (OMIM 602541) is characterized with early-onset hypotonia, muscle wasting, proximal weakness, cardiomyopathy, mildly elevated serum creatine kinase (CK) levels, and mild-to-moderate intellectual disability. We report two siblings in a consanguineous family admitted for psychomotor delay. Physical examination revealed proximal muscle weakness, contractures in the knee of elder sibling, diffuse mild generalized muscle atrophy, and dry skin with ichthyosis together with multiple nummular eczema in both siblings. Serum CK values were elevated up to 500 U/L. For genetic work-up, we performed whole exome sequencing (WES) after Nimblegen enrichment on the Illumina platform. The WES revealed a novel homozygous missense mutation in the Choline Kinase-Beta (CHKB) gene c.1031G>A (p.R344Q) in exon 9. Ichthyosis-like skin changes with intense pruritus and nummular eczema may lead to clinical diagnosis in cases with megaconial congenital muscular dystrophy.
\end{abstract}

\section{Introduction}

The congenital muscular dystrophies (CMD) are a group of diseases that show great clinical and genetic heterogeneity [1]. Megaconial congenital muscular dystrophy (OMIM 602541) is characterized with early-onset hypotonia, muscle wasting, proximal weakness, cardiomyopathy, mildly elevated serum creatine kinase levels, and mild-to-moderate intellectual disability [2]. Muscle biopsy shows dystrophic changes and mitochondrial abnormalities. The center of muscle fibers is usually depleted of mitochondria, but the mitochondria are markedly enlarged at the periphery [3]. The disease is caused by the loss-of-function mutations in choline kinasebeta $(C H K B)$. It is suggested that altered phospholipid composition in muscle mitochondrial membrane may lead to mitochondrial structural and functional abnormalities [4]. We report two siblings with megaconial congenital muscular dystrophy and the clinical relevance of skin changes in the differential diagnosis among other congenital muscular dystrophy subtypes.

\section{Patients and Genetic Analysis}

2.1. Patients. Informed consent was obtained from the parents for genetic investigations, recording and publishing the disease-related information. Two siblings aged three and eight-years-old were admitted for the investigation of psychomotor delay. Both of them were born at term without any complication. The parents were first degree consanguineous. Hypotonia from birth and psychomotor delay were noted. At age of 12 months, they were able to sit without support, but they could never gain the ability to stand and walk. Language delay and fine motor difficulties, which were more prominent in the younger sibling, became evident at the age of 24 months. At the age of 8 years, the elder sibling was able to speak fluently, but the younger sibling had only a few words at the age of 3 years. The elder sibling also learned to read, but was attending to school for special needs, and the Wechsler Intelligence Scale for Children revealed a total intelligence quotient score of 60 . They did not have a history of seizures. Physical examination revealed hypoactive deep 


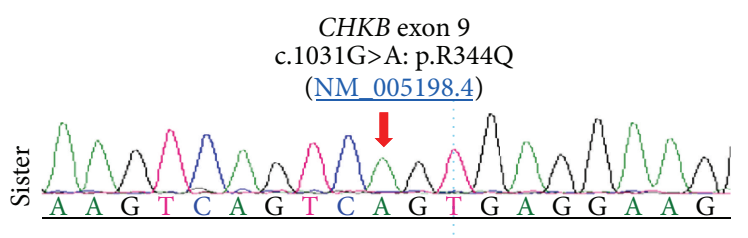

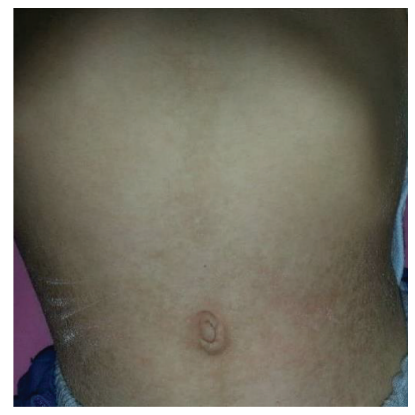

(a)

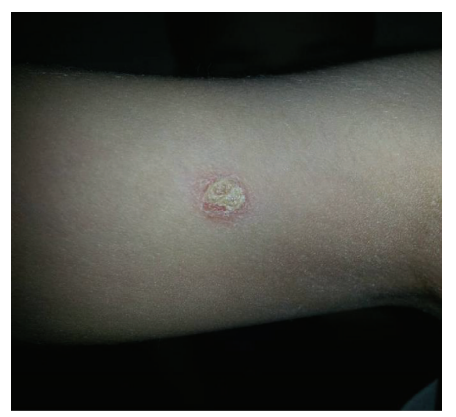

(b)
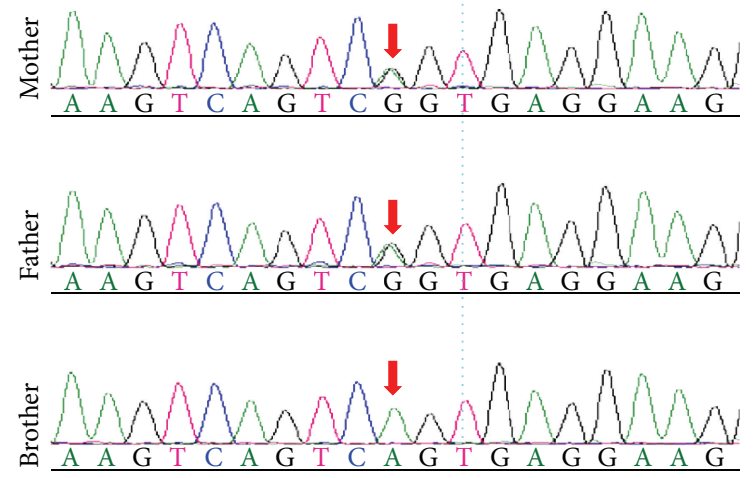

(c)

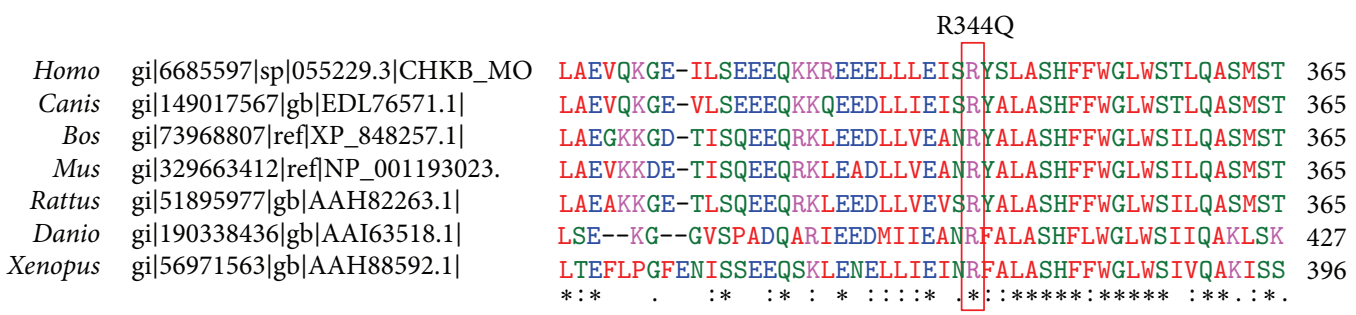

(d)

Figure 1: (a) Dry skin with ichthyosis in the younger sibling at the age of 3 years. (b) Dry skin with nummular eczema in the elder sibling at the age of 8 years. (c) Sanger confirmation of the mutation. Both affected siblings are homozygous and both parents are heterozygous carriers for R344Q mutation. (d) The conservation of the homozygous missense mutation within the vertebrates (http://www.ebi.ac.uk/Tools/msa/clustalw2/).

tendon reflexes, proximal muscle weakness, contractures in the knee of elder sibling, diffuse mild generalized muscle atrophy, dry skin with ichthyosis and multiple nummular eczema in the younger and elder siblings, respectively (Figures 1(a) and 1(b)). Brain and spinal cord magnetic resonance imaging and metabolic investigations including urine and serum amino acids and urine organic acids were normal. Serum creatine kinase values in the younger and elder siblings were $406 \mathrm{IU} / \mathrm{L}$ and $520 \mathrm{IU} / \mathrm{L}$, respectively. Nerve conduction studies were normal but needle examination showed myopathic potentials in all examined muscles. Muscle biopsy of the younger sibling at the age of 2.5 years revealed variation in fiber size, increased number of internal nuclei and fatty infiltration. Oxidative enzyme reactions (NADH-TR and SDH) showed enlarged mitochondria at the periphery of most fibers (data not shown). Immunolabelling of $\beta$-spectrin, dystrophin, dystroglycans ( $\alpha$ and $\beta$ subunits), calpain, sarcoglycans ( $\alpha, \beta, \gamma$, and $\delta$ subunits), collagen 6 , merosin/laminin-2, emerin, myotilin, and laminin A/C was normal (data not shown).
2.2. Exome Sequencing. For further genetic analysis, we performed whole exome sequencing (WES) from peripheral blood DNA of the younger male sibling after Nimblegen enrichment (SeqCap EZ Human Exome Library v2.00) on the Illumina HiSeq 2000 platform with $2 \times 100 \mathrm{bp}$ according to manufacturer instructions. The coverage was 75-fold, that is, 10x coverage for $96 \%$ of target sequences and $30 \mathrm{x}$ coverage for $86.2 \%$ of target sequences. The Cologne Center for Genomics VARBANK pipeline v.2.12 (https://varbank.ccg.uni-koeln.de/) used for data analysis for rare autosomal recessive disease in a consanguineous family revealed an unpublished novel homozygous missense variant in the Choline Kinase-Beta $(\mathrm{CHKB})$ gene c.1031G $>\mathrm{A}$ in exon 9 causing p.R344Q according to NM_005198.4 (Figure 1(c)). This variant was not present in the 1000 genomes (http://browser.1000genomes .org/index.html) or Exome Variant Server (http://evs.gs.washington.edu/EVS/) databases, but one allele was detected in 1/66650 European population chromosomes by the Exome Aggregation Consortium (http://exac.broadinstitute.org/). Arginine at 344 is highly conserved on vertebrates (Figure $1(\mathrm{~d})$ ) and analysis of this variant predicted it to be disease 
causing by MutationTaster (http://www.mutationtaster.org). The bioinformatics analysis shows that aberrant amino acid change is located on the choline kinase-motif domain of the protein [5]. The R344Q variant was confirmed by Sanger sequencing, which also revealed the homozygosity for the other affected sibling and carrier heterozygosity for the parents (Figure $1(\mathrm{c})$ ).

\section{Discussion}

Megaconial congenital muscular dystrophy (CMD) is characterized with early-onset hypotonia, muscle wasting, proximal weakness, cardiomyopathy, mildly elevated serum creatine kinase levels, and mild-to-moderate intellectual disability. The disease is caused by the loss-of-function mutations in choline kinase-beta $(C H K B)[3]$.

In the review of Mitsuhashi and Nishino [6], ichthyosislike skin changes have been observed in six patients out of 19. Five of them were of Turkish origin and one of them was of British origin. Ichthyosis-like skin changes were also reported by Quinlivan et al. in two unrelated patients with CMD who were shown to have one frameshift (W284*) and one missense (N241S) homozygous mutation in $\mathrm{CHKB}$, respectively [7]. In those cases, ichthyosis in proximal areas with fine scaling was suggested as an important clue to the diagnosis. The largest megaconial CMD patient series by Haliloglu et al. demonstrated that ichthyosis-like changes was the most frequent (11/15) skin change in megaconial CMD [8]. Nummular eczema, which is shown in the elder sister of our patients, has not been described before and the symptoms improved after topical corticosteroid therapy.

Examination of skin is very important in patients with suspected congenital muscular dystrophy. Patients with collagen VI related dystrophies including Ullrich CMD and Bethlem myopathy have keloid or atrophic scar formation, striae, thin skin, soft skin on palms and soles, and hyperkeratosis pilaris [1]. Another severe LGMD type presenting with skin lesions together with congenital muscular dystrophy, myasthenic symptoms, and epidermolysis bullosa simplex is caused by plectin (PLEC1) mutations and should be considered in the different diagnosis [9].

\section{Conclusion}

Patients with megaconial congenital muscular dystrophy have ichthyosis-like skin changes with intense pruritus, which will lead to clinical diagnosis in cases with congenital muscular dystrophy. Nummular eczema may also be a new skin finding in megaconial congenital dystrophy, which warrants further reports.

\section{Consent}

The study was approved by the institutional review board of the Ethics Committee of the University Hospital of Cologne. Informed consent for research and publication was obtained from both the patients and the family.

\section{Competing Interests}

The authors declare that they have no conflict of interests.

\section{Acknowledgments}

The authors would like to thank the patients and the family for their enrollment. This work was supported by the Deutsche Forschungsgemeinschaft Emmy Noether Grant (CI 218/1-1) and Muscular Dystrophy Association (Developmental Grant Cirak).

\section{References}

[1] C. G. Bönnemann, C. H. Wang, S. Quijano-Roy et al., "Diagnostic approach to the congenital muscular dystrophies," Neuromuscular Disorders, vol. 24, no. 4, pp. 289-311, 2014.

[2] I. Nishino, O. Kobayashi, Y.-I. Goto et al., "A new congenital muscular dystrophy with mitochondrial structural abnormalities," Muscle \& Nerve, vol. 21, no. 1, pp. 40-47, 1998.

[3] S. Mitsuhashi, A. Ohkuma, B. Talim et al., "A congenital muscular dystrophy with mitochondrial structural abnormalities caused by defective de novo phosphatidylcholine biosynthesis," American Journal of Human Genetics, vol. 88, no. 6, pp. 845-851, 2011.

[4] S. Mitsuhashi, H. Hatakeyama, M. Karahashi et al., "Muscle choline kinase beta defect causes mitochondrial dysfunction and increased mitophagy," Human Molecular Genetics, vol. 20, no. 19, Article ID ddr305, pp. 3841-3851, 2011.

[5] E. Malito, N. Sekulic, W. C. S. Too, M. Konrad, and A. Lavie, "Elucidation of human choline kinase crystal structures in complex with the products ADP or phosphocholine," Journal of Molecular Biology, vol. 364, no. 2, pp. 136-151, 2006.

[6] S. Mitsuhashi and I. Nishino, "Megaconial congenital muscular dystrophy due to loss-of-function mutations in choline kinase $\beta$," Current Opinion in Neurology, vol. 26, no. 5, pp. 536-543, 2013.

[7] R. Quinlivan, S. Mitsuahashi, C. Sewry et al., "Muscular dystrophy with large mitochondria associated with mutations in the CHKB gene in three British patients: extending the clinical and pathological phenotype," Neuromuscular Disorders, vol. 23, no. 7, pp. 549-556, 2013.

[8] G. Haliloglu, B. Talim, C. G. Sel, and H. Topaloglu, "Clinical characteristics of megaconial congenital muscular dystrophy due to choline kinase beta gene defects in a series of 15 patients," Journal of Inherited Metabolic Disease, vol. 38, no. 6, pp. 10991108, 2015.

[9] K. Forrest, J. E. Mellerio, S. Robb et al., "Congenital muscular dystrophy, myasthenic symptoms and epidermolysis bullosa simplex (EBS) associated with mutations in the PLEC1 gene encoding plectin," Neuromuscular Disorders, vol. 20, no. 11, pp. 709-711, 2010. 

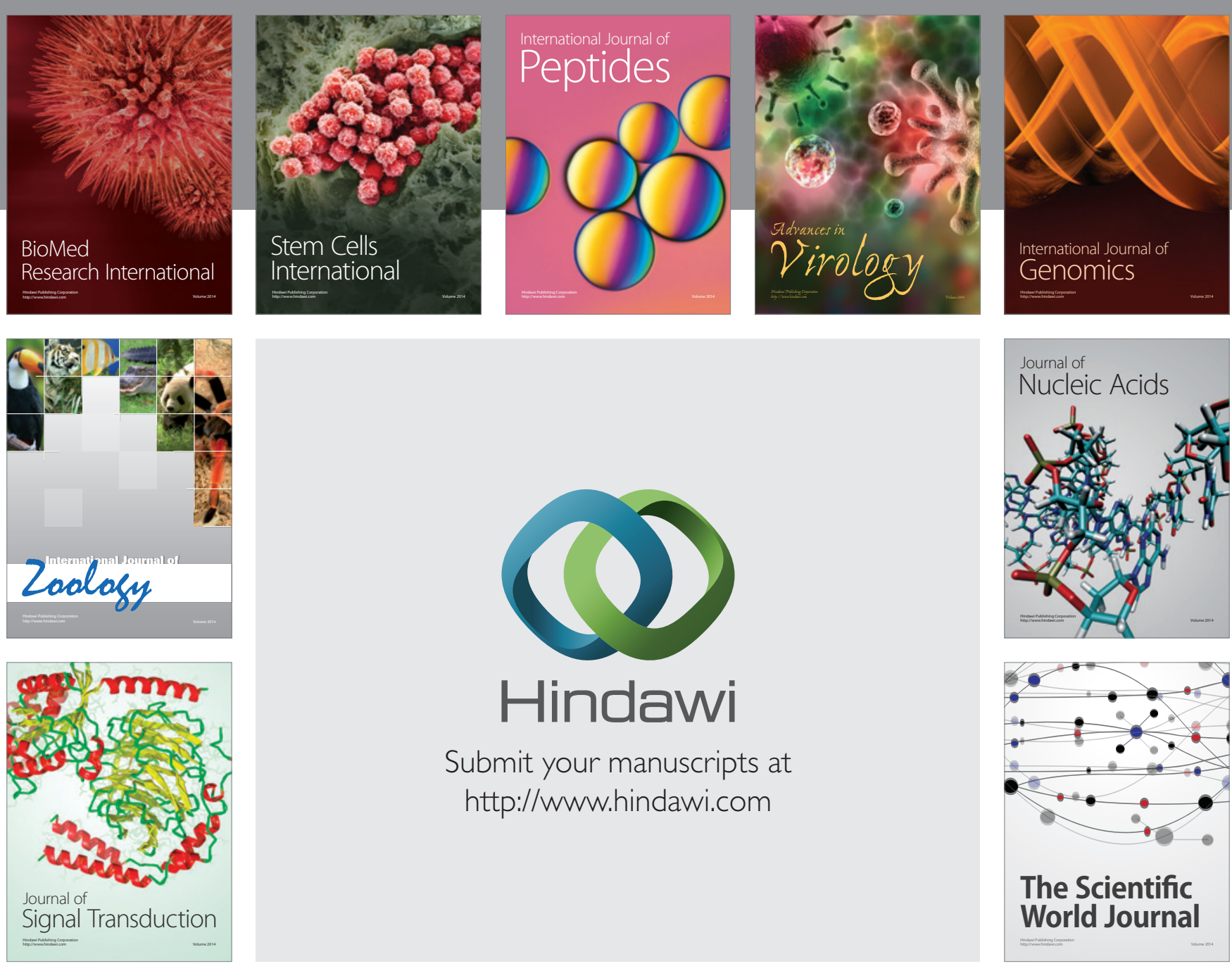

Submit your manuscripts at

http://www.hindawi.com
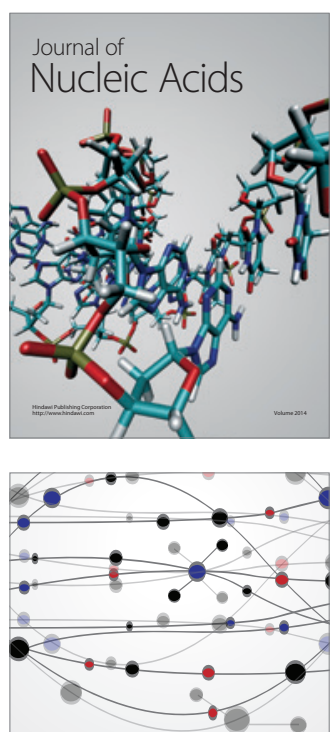

The Scientific World Journal
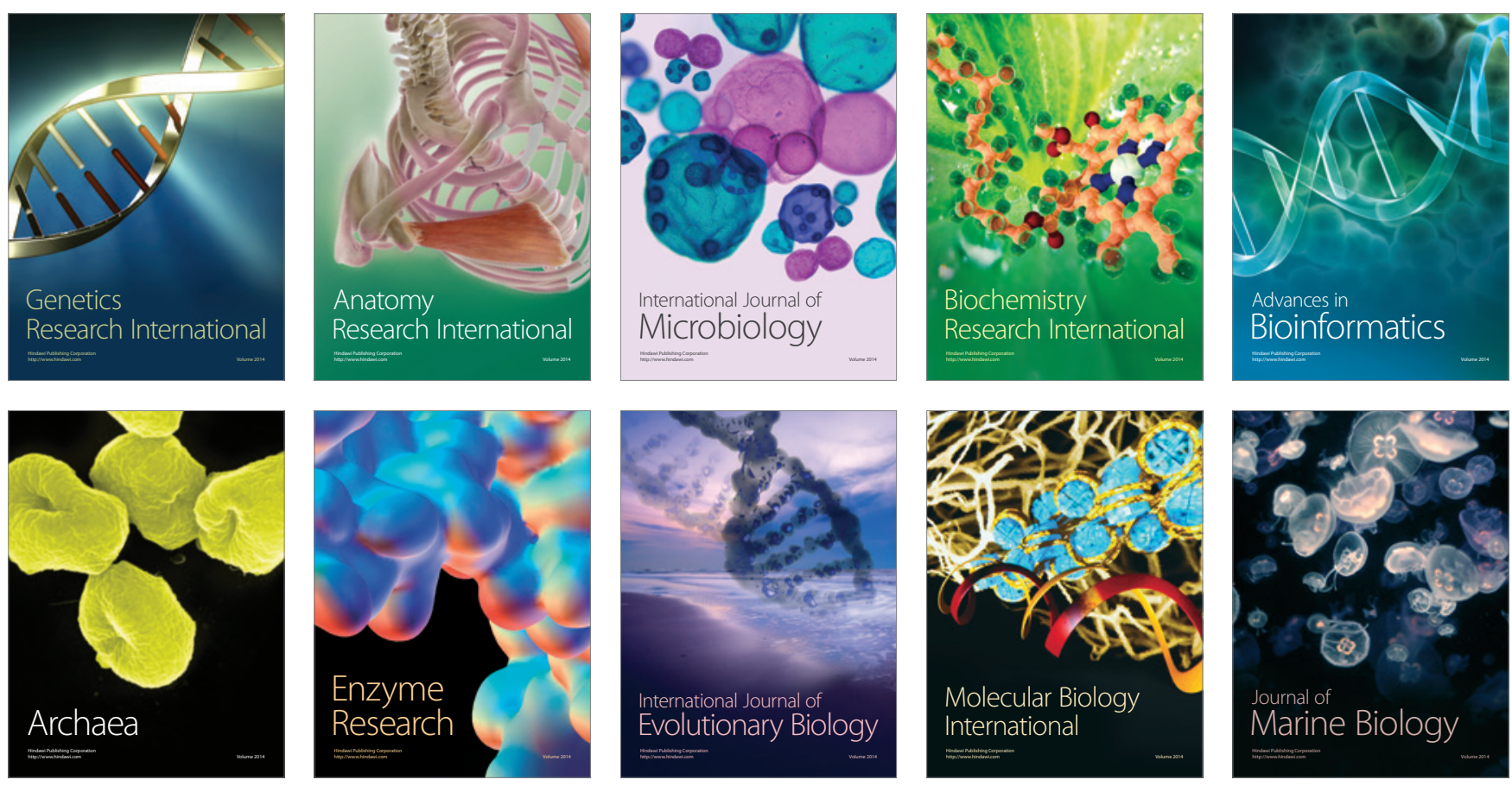